\title{
Effect of Local Injection of Platelet Rich Plasma on Repair of Rats' Ruptured Achilles Tendon Tissue
}

\author{
Lei Zhang ${ }^{1}$, Xinhua Cheng ${ }^{2, *}$ \\ ${ }^{1}$ Department of Bone and Joint Surgery, Renmin Hospital, Hubei University of Medicine, Shiyan \\ Renmin Hospital, Shiyan, Hubei Province, China,442000 \\ ${ }^{2}$ Department of Microscopic Orthopaedic, Renmin Hospital, Hubei University of Medicine, Shiyan \\ Renmin Hospital, Shiyan, Hubei Province, China,442000 \\ *Corresponding author, E-mail: hubeiwenbo@163.com \\ Address: Chaoyang District Road No. 39, Shiyan City, Hubei Prov,P.R.China,442000
}

Keywords: rat; rupture of achilles tendon; platelet rich plasma; tissue repair

Abstract: Objective: to discuss the effect of local injection of platelet rich plasma on repair of rats' ruptured achilles tendon tissue. Method: 80 rats with ruptured achilles tendon were chosen in this study, and they were classified into two groups with double blind method. The control group (40 rats) was not treated, while platelet rich plasma was locally injected for the observation group (40 rats). The repair of ruptured achilles tendon tissue in both groups was compared and analyzed. Results: the grading of tendon adhesion degree in both groups presented exacerbation $1-4 \mathrm{w}$ after the treatment, $\mathrm{P}>0.05$. At the rupture of achilles tendon of rats in the observation group, the improvement degree of positive density value of collagenous fiber I was superior to the control group. In the biomechanics test, the elasticity modulus and maximum tensile strength were superior to the control group, $\mathrm{P}<$ 0.05 . The maximum sliding distance of both groups had no difference, $\mathrm{P}>0.05$. Conclusion: local injection of platelet rich plasma for the rats after the rupture of achilles tendon has the obvious effect, and it can accelerate achilles tendon tissue healing.

Human standing up, running and jumping are closely related to the functions of achilles tendon which is the thickest and strongest muscle tendon ${ }^{11]}$. After the rupture of achilles tendon, claudication (one side) or the inability to walk (both sides) will be cussed. There are many methods to clinically treat this disease. Usually, conservative treatment or operative treatment may be chosen. Although corresponding treatment effect can be gained, as medical level in China continues to rise, more scholars consider tissue repair promotion should be concerned during the treatment of rupture of achilles tendon to improve the prognosis effect ${ }^{[2]}$. To further improve the repair effect of ruptured achilles tendon tissue, 80 rats with ruptured achilles tendon tissue were classified into two groups with double blind method, and different treatment methods were given for the two groups to study the repair effect of ruptured achilles tendon tissue after the rats received local injection of platelet rich plasma. 


\section{Data and Method}

\subsection{General data}

In this study, 80 rats with ruptured achilles tendon tissue were chosen as the object of study. They were classified into two groups with double blind method. Among the 40 rats in the control group, there were 23 male rats and 17 female rats, with the weight of 190-235kg and mean value of $(209.64 \pm 10.78) \mathrm{kg}$. Among the 40 rats in the observation group, there were 21 male rats and 19 female rats, with the weight of $193-240 \mathrm{~kg}$ and mean value of $(213.17 \pm 10.03) \mathrm{kg}$. The baseline data of both groups had no difference, $\mathrm{P}>0.05$.

\subsection{Method}

Intraperitoneal injection of 3\% pentobarbital $(0.1 \mathrm{ml} / 100 \mathrm{~g})$ was conducted for the rats in both groups. The achilles tendon and calcaneus at both sides of rats were crosswise cut for $7 \mathrm{~mm}$, and cut off after achilles tendon was exposed. About 2mm-long coloboma was prepared. After modeling, platelet rich plasma was injected around the rupture end of achilles tendon in the observation group, and local swell was observed. 100ul platelet rich plasma was injected for achilles tendon in each side. The rats in the control group were not treated. After the wound was rinsed thoroughly, 4-0 suture was used to suture the wound ${ }^{[3]}$. The rats in the observation group received local injection of 100ul platelet rich plasma once per week. The effect was observed after $4 \mathrm{~W}$. $3 \mathrm{~d}$ after the first injection, penicillin was used for the rats every day for anti-infection ${ }^{[4]}$.

\subsection{Observation indexes}

Tendon adhesion grading of both groups was observed. The newborn achilles tendon tissues at $2 \mathrm{~mm}$ of both-side achilles tendon rupture 1, 2, 3, and $4 \mathrm{~W}$ after the operation were taken for immunohistochemistrical dyeing and biomechanics test. Survival, activity, and incision infection rate of both groups were observed.

\subsection{Evaluation criteria}

Evaluation of rats' achilles tendon healing and tendon adhesion: Grade I means no adhesion; Grade II means thin film adhesion exists; Grade III means loose adhesion exists; Grade IV means moderate compact adhesion exists, and different degrees of one drop of blood could be seen; Grade $\mathrm{V}$ means compact adhesion exists, and the separation difficulty is very larg $\mathrm{e}^{[5]}$.

\subsection{Statistical method}

SPSS 20.0 statistics software was used to analyze the clinical data. The measurement data were expressed with ( $x \pm s$ ), and tested with t test. The enumeration data were expressed with (\%), and tested with chi-square test. $\mathrm{P}<0.05$ means there is statistical significance.

\section{Results}

\subsection{Grading of tendon adhesion degree}

After the treatment, tendon adhesion degree of both groups showed exacerbation, $\mathrm{P}>0.05$, as shown in Tab.1. 
Tab.1 Grading of tendon adhesion degree $(\%, \mathrm{n})$

\begin{tabular}{ccccccc}
\hline Time & Group & I & II & III & IV & V \\
\hline Before & $\begin{array}{c}\text { Observatio } \\
\text { n group }\end{array}$ & 2 & 4 & 0 & 0 & 0 \\
treatment & $\begin{array}{c}\text { Control } \\
\text { group }\end{array}$ & 3 & 2 & 0 & 0 & 0 \\
t value & - & & 0.1054 & & \\
P value & - & & 0.7454 & & 4 \\
& Observatio & 0 & 3 & 4 & 3 & 3 \\
After & n group & & & 3 & 3 & \\
treatment & Control & 0 & 2 & 0.5236 & & \\
group & - & & & 0.4693 & & \\
P value & - & & & & & \\
\hline
\end{tabular}

\subsection{Positive density of collagenous fiber I}

Before the treatment, the comparison of positive density value of collagenous fiber I at the rupture of achilles tendon in both groups had no difference, $\mathrm{P}>0.05$. After the treatment, the improvement degree of positive density value of collagenous fiber I at the rupture of achilles tendon in the observation group was superior to the control group, $\mathrm{P}<0.05$, as shown in Tab.2.

Tab.2 Positive density of collagenous fiber I $(x \pm s)$

\begin{tabular}{ccccc}
\hline Group & $1 \mathrm{~W}$ & $2 \mathrm{~W}$ & $3 \mathrm{~W}$ & $4 \mathrm{~W}$ \\
\hline $\begin{array}{c}\text { Observation } \\
\text { group }\end{array}$ & $178.60 \pm 0.71$ & $189.74 \pm 2.15$ & $192.03 \pm 2.70$ & $194.07 \pm 1.35$ \\
Control & $179.43 \pm 0.65$ & $180.09 \pm 3.66$ & $182.68 \pm 3.15$ & $181.97 \pm 3.62$ \\
group & 5.4533 & 14.3781 & 14.2534 & 19.8075 \\
t value & 0.0417 & 0.0153 & 0.0112 & 0.0041 \\
P value & & & & \\
\hline
\end{tabular}

\subsection{Biomechanics test}

In the biomechanics test for the rats in the observation group, the elasticity modulus and maximum tensile strength were superior to the control group, $\mathrm{P}<0.05$. The maximum sliding distance of both groups had no difference, $\mathrm{P}>0.05$, as shown in Tab.3.

Tab.3 Biomechanics test $(x \pm s)$

\begin{tabular}{|c|c|c|c|}
\hline Group & $\begin{array}{l}\text { maximum sliding } \\
\text { distance (mm) }\end{array}$ & Elasticity modulus (MPa) & $\begin{array}{l}\text { Maximum tensile strength } \\
(\mathrm{N})\end{array}$ \\
\hline $\begin{array}{l}\text { Observatio } \\
\text { n group }\end{array}$ & $5.97 \pm 1.06$ & $8.61 \pm 2.34$ & $35.68 \pm 5.14$ \\
\hline $\begin{array}{l}\text { Control } \\
\text { group }\end{array}$ & $5.10 \pm 1.53$ & $1.79 \pm 1.48$ & $13.20 \pm 4.62$ \\
\hline t value & 2.9562 & 15.5787 & 20.5720 \\
\hline $\mathrm{P}$ value & 0.7134 & 0.0231 & 0.0019 \\
\hline
\end{tabular}




\section{Discussion}

The rupture of achilles tendon is mostly caused by strenuous exercise injury. Usually, conservative treatment or operative treatment is chosen to treat this disease. Conservative treatment guarantees the ankle joint presents the plantar flexion position, and then plaster immobilization is applied for $4 \mathrm{~W}$ to make the rupture of achilles tendon fully contact and heal. Later, orthosis is used to fix for $4 \mathrm{~W}$. Operative treatment aims to suture both ends of rupture of achilles tendon, and the suture is used to make the rupture ends contact fully and firmly. Then, plaster immobilization is applied for $4 \mathrm{~W}$ to make the rupture of achilles tendon heal ${ }^{[6]}$. With rapid development of medical career in recent years, more medical instruments and medicines are researched, development and applied extensively. To further improve clinical treatment effect of rupture of achilles tendon, many medical experts have conducted more in-depth researches on the pathogenesis and treatment scheme of this disease. More adjuvant therapies also appear, such as local injection of platelet rich plasma. Multiple prospective study results show this method has obvious clinical effect on adjuvant therapy of rupture of achilles tendon ${ }^{[8]}$.

zhu Junshan, Feng Xiuzhen and Zhuang Rujie ${ }^{[9]}$ summarized research progress on treatment of ankle-related diseases with platelet rich plasma, and the researches showed that platelet rich plasma could enhance the treatment effect for the patients with acute rupture of achilles tendon receiving improved Krackow sutura. Besides, patients' isokinetic muscle strength, ankle joint motion range, crus leg circumference and Leppilahti achilles tendon repair scores improved obviously 2, 6, 12 and 24M after the operation. large differences existed, compared with the control group, $\mathrm{P}<0.05$. Zhang Bo, Wei Bingdan and Gan Kunming et al. ${ }^{[10]}$ indicated that, platelet rich plasma is a product which contains high-concentration blood platelet prepared by autoblood. Platelet rich plasma contains concentrated blood platelet, a small quantity of white blood cells and fibrous protein. After thrombin/calcium chloride excitation, degranulation effect will appear, and multiple growth factors will be released, such as platelet-derived growth factor and epidermal growth factor. They can obviously promote tissue repair effect. Therefore, platelet rich plasma is widely applied in clinical treatment of muscle tendon, ligament, cartilage or muscle injury. The research results show that, the grading of tendon adhesion degree in both groups presented exacerbation after the treatment. At the rupture of achilles tendon of rats in the observation group, the improvement degree of positive density value of collagenous fiber I was superior to the control group. In the biomechanics test, the elasticity modulus and maximum tensile strength were superior to the control group, $\mathrm{P}<0.05$. The maximum sliding distance of both groups had no difference, $\mathrm{P}>0.05$.

In conclusion, local injection of platelet rich plasma has obvious repair effect on rats' ruptured achilles tendon tissue, and thus has very excellent clinical promotion value.

\section{References}

[1] Ahmad HS, Farrag SE, Okasha AE, Kadry AO, Ata TB, Monir AA, Shady I.Clinical outcomes are associated with changes in ultrasonographic structural appearance afterplatelet-rich plasma treatment for knee osteoarthritis.Int $J$ Rheum Dis. 2018 May;21(5):960-966.

[2] Zhang H, Bai Y, Liu C, Jin S, Su K, Liu Y, Lü Z.Effect of intra-articular injection of platelet-rich plasma on interleukin-17 expression in synovial fluid and venous plasma of knee osteoarthritis patients.Zhongguo Xiu Fu Chong Jian Wai Ke Za Zhi. 2017 Aug 1;31(8):918-921.

[3] Yu T, Pan H, Hu Y, Tao H, Wang K, Zhang C.Autologous platelet-rich plasma induces bone formation of tissue-engineered bone with bone marrow mesenchymal stem cells on beta-tricalcium phosphate ceramics.J Orthop Surg Res. 2017 Nov 21;12(1):178.

[4] Song Y, Lin K, He S, Wang C, Zhang S, Li D, Wang J, Cao T, Bi L, Pei G.Nano-biphasic calcium phosphate/polyvinyl alcohol composites with enhanced bioactivity for bonerepair via low-temperature three-dimensional printing and loading with platelet-rich fibrin.Int J Nanomedicine. 2018 Jan 25;13:505-523. 
[5] Wittig O, Romano E, González C, Diaz-Solano D, Marquez ME, Tovar P, Aoun R, Cardier JE.A method of treatment for nonunion after fractures using mesenchymal stromal cells loaded on collagen microspheres and incorporated into platelet-rich plasma clots.Int Orthop. 2016 May;40(5):1033-8.

[6] Beigi Mohammad-Hossein,Atefi Atefeh,Ghanaei Hamid-Reza,etc.Activated platelet-rich plasma (PRP) improves cartilage regeneration using adipose stem cells encapsulated in a 3D alginate scaffold.[J].Journal of tissue engineering and regenerative medicine, 2018.

[7] Zhang HF, Wang CG, Li H, Huang YT, Li ZJ.ntra-articular platelet-rich plasma versus hyaluronic acid in the treatment of knee osteoarthritis: a meta-analysis.Drug Des Devel Ther. 2018 Mar 5;12:445-453.

[8] Geburek Florian,Gaus Moritz,van Schie Hans T M,etc.Effect of intralesional platelet-rich plasma (PRP) treatment on clinical and ultrasonographic parameters in equine naturally occurring superficial digital flexor tendinopathies - $a$ randomized prospective controlled clinical trial.[J].BMC veterinary research,2016,12(1).

[9] Fukawa T, Yamaguchi S, Akatsu Y, Yamamoto Y, Akagi R, Sasho T.Safety and Efficacy of Intra-articular Injection of Platelet-Rich Plasma in Patients With AnkleOsteoarthritis.Foot Ankle Int. 2017 Jun;38(6):596-604.

[10] Tao H, Zhang C, Zeng B, Yuan T, Xu J, Song W.Experimental study on the treatment of femur head necrosis with tricalcium phosphate andplatelet-rich plasma].Zhongguo Xiu Fu Chong Jian Wai Ke Za Zhi. 2005 Mar 15;19(3):170-3. Chinese. 